\title{
Compassionate and Respectful Maternity Care and Associated Factors among Women Attending Delivery Services at Public Health Facilities of Hawassa City, Southern Ethiopia
}

\author{
Bersabeh Bedru Wochefu', Abdela Amano Abdo ${ }^{2}$ and Dubale Dulla Koboto ${ }^{3 *}$ \\ ${ }^{1}$ Disease Control and Prevention Department, Regional Health Bureau, Southern Nation, Nationalities and \\ Peoples Region, Ethiopia \\ ${ }^{2}$ School of Public Health, College of Medicine and Health Science, Hawassa University, Ethiopia \\ ${ }^{3}$ Department of Midwifery, College of Medicine and Health Science, Hawassa University, Ethiopia
}

*Corresponding author: Dubale Dulla Koboto, Department of Midwifery, College of Medicine and Health Science, Hawassa University, Southern Nation, Nationalities and Peoples Region, Hawassa, Ethiopia, Tel +2519-1385-0895

\begin{abstract}
Background: Providing compassionate and respectful maternity care service to laboring mothers is one of the most important interventions to ensure the survival of women. However, compassionate and respectful maternity care has received less attention both in practice and research.

Objective: To assess the compassionate and respectful maternity care and associated factors among women attending delivery services at the Public health Facilities of Hawassa City, Southern Ethiopia.

Methods: Institution based cross-sectional study design was conducted to collect data from study participants in Hawassa City from April to May 2019. Study subjects were selected through systematic random sampling based on their proportional distribution of sample size to each institution. A structured questionnaire was used to collect the data. The data was coded and entered into Epi data version 3.1 and the analysis was carried out in SPSS versions 22. Bivariable and Multivariable binary logistic regression analysis with $95 \% \mathrm{Cl}$ was carried out.

Result: The prevalence of disrespect and abuse during childbirth was $63.5 \%$ (95\% Cl: 60.2-67.2). Disrespect and abuse were more common in women who had no formal education $(\mathrm{AOR}=1.64 ; 95 \% \mathrm{Cl}: 1.07,2.53)$ and Primary (18) education ( $\mathrm{AOR}=2.63 ; 95 \% \mathrm{Cl}: 1.69,4.08$ ) as compared with Secondary and above. Women who had less than 4 ANC visit for current pregnancy, (AOR $=1.75 ; 95 \% \mathrm{Cl}$ : 1.26 , $2.43)$, Spontaneous vaginal delivery (SVD), $(A O R=2.347$; $95 \% \mathrm{Cl}: 1.482,3.716)$ and women who face complication during labor and delivery $(\mathrm{AOR}=1.598 ; 95 \% \mathrm{Cl}$ : 1.036 , 2.465) was associated with disrespect and abuse during childbirth.
\end{abstract}

Conclusions: The prevalence of disrespect and abuse during childbirth was high in this study area. A key implication of the finding of this is that efforts have to be taken to increase facility-based delivery by addressing determinant factors of disrespect and abuse to ensure utilization of quality institutional delivery care.

\section{Keywords}

Disrespect, Abuse, Delivery, CRMC, Hawassa, Southern Ethiopia

\section{Abbreviations and Acronyms}

A\&D: Abuse and Dis-Respective; ANC: Antenatal Care; BEmONC: Basic Emergency Obstetric Neonatal Care; $\mathrm{Cl}$ : Confidence Interval; $\mathrm{Cl}$ : Confidence Interval; CRMC: Compassionate Respectful Maternity Care; EDHS: Ethiopian Demographic and Health Survey; FMOH: Federal Minster of Health; HSTP: Health Sector Transformation Program; HURCSH: Hawassa University Referral Comprehensive Specialized Hospital; MDGs: Millennium Development Goals; MMR: Maternal Mortality Rate; PHCU: Primary Health Care Unit; SNNPR: Southern Nation Nationality People's Region; SPSS: Statistical Package for Social Science; SSA: Sub-Sahara Africa; TRA: Translating Research to Action; WHO: World Health Organization

\section{Background}

In 2015 more than 303,000 women died of easily preventable obstetric complications in the globe, of which $99 \%$ were from less developed countries. SubSaharan Africa alone accountable for $66 \%$ of the deaths

Citation: Wochefu BB, Abdo AA, Koboto DD (2021) Compassionate and Respectful Maternity Care and Associated Factors among Women Attending Delivery Services at Public Health Facilities of Hawassa City, Southern Ethiopia. Int Arch Nurs Health Care 7:165. doi.org/10.23937/2469-5823/1510165 Accepted: September 09, 2021: Published: September 11, 2021

Copyright: (C) 2021 Wochefu BB, et al. This is an open-access article distributed under the terms of the Creative Commons Attribution License, which permits unrestricted use, distribution, and reproduction in any medium, provided the original author and source are credited. 
[1]. Ethiopia is an African countries with one of the highest maternal mortality rates (MMR) of 412 maternal deaths per 100,000 live births [2].

Universal use of skilled birth attendants is the most effective means of reducing maternal mortality is healthcare facilities because the majority of pregnancyrelated complications cannot be predicted and require urgent and immediate attention $[3,4]$. A universal human right declares that every childbearing woman in every health system around the world should practice respect and compassionate care as part of their basic human rights, including respect for women's autonomy, dignity, feelings, and choices [5], because, respectful health care is the determining factor to increase client satisfaction and health-seeking behavior of the community [6].

World Health Organization (WHO) defines compassionate and respectful maternal care (CRMC) as the right every woman has to attain the highest standard of health which similarly includes the right to dignity compassionate and respectful health care to all childbearing women around the world throughout pregnancy, childbirth, and the postnatal period $[7,8]$.

Disrespectful and abuse during facility childbirth include physical abuse, lack of consent for care, nonconfidential care, undignified care, abandonment, discrimination, and detention in facilities for failure to pay user fees [9]. A study conducted on disrespect and abuse during facility-based childbirth in Ethiopia showed that $67.1 \%$ of women experience disrespect and abuse, and the most common abuses were physical abuse, non-consented care, and non-confidential care [10]. Another, studies conducted in different areas also showed that a significant number of women experience non-confidential care [11-13].

Several studies identified different factors associated with non-compassionated and disrespectful cares such as socio-demographic factors [12-18]; obstetric related factors $[11,19,20]$; social factors; normalization of disrespect and abuse, lack of engagement and oversight, financial barriers, lack of autonomy and empowerment [12,13]; institutional factors; lack of standardized infrastructure and lack of responsibility mechanisms, lack of resource and staff shortage [11,21$23]$ and providers related factors $[9,19,22-24]$.

In Ethiopia, though the federal ministry of health gives special attention to address gaps in the provision of compassionate and respectful health care in the health system $[8,25]$, still there is a breach in the implementation of CRMC. Besides, there were also limited researches conducted to identify different factors associated with non-compassionated and disrespectful maternity care in Ethiopia.

Hence, the aim of this study was to assess the prevalence of compassionated and respectful maternity care and its associated factors among women attending delivery services at public health facility of Hawassa City, Southern Ethiopia.

\section{Methods and Materials}

\section{Study setting}

Hawassa is the capital city of Southern Nation Nationalities and Peoples Region and has 8 Sub City and is $273 \mathrm{~km}$ far from Addis Ababa, the capital city of Ethiopia. The city contains one comprehensive specialized hospital, one general hospital, one primary hospital and eleven health centers. The total population of the city is estimated to be 376,539 from which 191,281 are females and 5149 women gave birth to live children in the last six months.

\section{Study design and participants}

Intuitional based cross-sectional study was carried out among women who gave birth in public health facility of Hawassa City from April to May, 2019. The sample size was calculated using single population proportion formula, $\mathrm{n}=(\mathrm{Z} \alpha / 2)^{2} \times P(1-P) / \mathrm{d}^{2}$, considering the following assumptions: $95 \%$ confidence interval, prevalence of respectful maternity care experienced $57 \%$ [14]; margin of error 5\%, design effect of 2; and $10 \%$ for non-response and a total of 806 women were included in the study.

Two stage sampling technique was used to select the study participants. First, five health centers were selected from 11 health centers using simple random sampling technique and all hospitals were selected because of level difference. Then the woman who gave birth at the selected health facility were selected using systematic random sampling technique. The sampling interval was calculated based on the number of women who gave birth in these facilities in six months of previous year. Finally, the study participants were selected from each health facility using " $k$ " value interval $(K / n) 1716 / 830 \approx 2$.

\section{Study variables}

The main outcome variable was compassionate and respectful maternity care and it was measured based on seven components of CRMC. Each CRMC components were summarized by adding constituent questions coded by yes (1) and no (0). Then components were recoded to yes (1) when fully achieved or no (0) when any of questions was responded "no". Finally, CRMC was computed by adding individual components and scores of more zero were considered yes (1) that indicate presence of abuse and disrespectful care and value summarized to zero (0) indicates no abuse and disrespectful care.

The explanatory variables includes; socio demographic factors (age, marital status, religion, education, socio economic back ground), Obstetric 
related factors (parity, gravity, place of delivery, length of stay in the health facility), health system related factors (lack of standardized infrastructure, lack of responsibility mechanisms, lack of resource and staff shortage), and individual related factor (normalization of disrespect and abuse).

\section{Data collection procedures}

Data were collected using interviewer administered questionnaire by nurses who were not working at the selected health facilities and supervised by mater holder public health professionals. Seven nurses and two supervisors were involved in data collection process. Training was given for both data collectors and supervisors on how to collect the data. The content and consistency of the data was checked by the investigators on daily base.

\section{Data processing and analysis}

All the collected data were entered using epi data version 3.1 and analyzed using the statistical package for social sciences (SPSS) for windows version 20. Descriptive statistics with frequencies and percentages for categorical data, as well as means and standard deviation for numerical data, were used to summarize the data. Bi-variable and multivariable binary logistic regression was used to identify factors associated with CRMC. A p-value of $<0.2$ was used as a cut of point in bi-variable analysis to select the candidate variables for multivariable analysis. Adjusted odds ratio (AOR) with $95 \% \mathrm{Cl}$ was used to determine association.

\section{Ethical clearance}

The study was approved by the Institutional Review Board of Pharma College of Health Science Hawassa campus. A formal letter of cooperation was delivered to the study area (Health Department, Hawassa city administration). Participants were clearly informed of the general purpose, possible risks, and benefits of the study and verbal consent was obtained from each participants before the data collection. The issue of confidentiality and privacy of participants" was secured by coding the data in terms of anonymity.

\section{Results}

\section{Socio-demographic characteristics of respondents}

A total of 806 women who gave birth in public health facility of Hawassa City participated in the study with a response rate of $97.11 \%$. Nearly half, $(44.0 \%)$, of the participants were from HURCSH; 324 (40.2\%) were in the age range of $25-34$ years with mean ( \pm SD) age of 30.1 ( \pm 7.44 ) years; $90.0 \%$ were married; while, only $88(10.9 \%)$ were college and above education. About 163 (20.2\%) were government employee with average monthly income of 1630 ETB (\$48) (Table 1).

\section{Obstetric and personal related factors}

As provided in Table 2, more than one third (37.1\%)

Table 1: Socio-demographic characters of women who gave birth in public health facility of Hawassa City, Southern Ethiopia, 2019.

\begin{tabular}{|c|c|c|c|}
\hline Variables & Category & Frequency & $(\%)$ \\
\hline \multirow[t]{3}{*}{ Age of mother in years } & $15-24$ & 223 & $(27.7)$ \\
\hline & $25-34$ & 324 & $(40.2)$ \\
\hline & 35 and above & 259 & $(32.1)$ \\
\hline \multirow[t]{4}{*}{ Marital status } & Single & 32 & $(4.0)$ \\
\hline & Married & 725 & $(90.0)$ \\
\hline & Divorced & 34 & $(4.2)$ \\
\hline & Widowed & 15 & $(1.9)$ \\
\hline \multirow[t]{5}{*}{ Mothers level of education } & No formal education & 136 & $(16.9)$ \\
\hline & Read and write & 118 & $(14.6)$ \\
\hline & Primary (1-8) & 338 & $(41.9)$ \\
\hline & Secondary (9-12) & 126 & $(15.6)$ \\
\hline & College and above & 88 & $(10.9)$ \\
\hline \multirow[t]{6}{*}{ Mothers occupation } & House wife & 323 & $(40.1)$ \\
\hline & Private employee & 163 & $(20.2)$ \\
\hline & Government employee & 88 & $(10.9)$ \\
\hline & Merchant & 172 & $(21.3)$ \\
\hline & Student & 50 & $(6.2)$ \\
\hline & Others & 10 & $(1.2)$ \\
\hline \multirow[t]{3}{*}{ Family monthly income (ETB) } & $<1000$ ETB & 201 & $(24.9)$ \\
\hline & 1000-2575 ETB & 471 & $(58.4)$ \\
\hline & $>2575$ ETB & 134 & $(16.6)$ \\
\hline
\end{tabular}


Table 2: Obstetric related factors of women who gave birth in public health facility of Hawassa City, Southern Ethiopia, 2019.

\begin{tabular}{|c|c|c|}
\hline Variables & Category & Frequency (\%) \\
\hline \multirow[t]{2}{*}{ ANC for this child } & Yes & $583(72.3)$ \\
\hline & No & $223(27.7)$ \\
\hline \multirow[t]{5}{*}{ Whom did you seen } & Physician & $142(24.4)$ \\
\hline & Nurse & $38(6.5)$ \\
\hline & Midwifery & $324(55.6)$ \\
\hline & HEWs & $71(12.2)$ \\
\hline & Health officers & $8(1.4)$ \\
\hline \multirow[t]{3}{*}{ Place of current ANC } & Home & $86(14.8)$ \\
\hline & Health center & $486(83.4)$ \\
\hline & hospital & $11(1.9)$ \\
\hline \multirow[t]{2}{*}{ No of ANC visit for current pregnancy } & $<4$ & $512(63.5)$ \\
\hline & $\geq 4$ & $294(36.5)$ \\
\hline \multirow[t]{4}{*}{ Parity } & Nullipara & $100(12.4)$ \\
\hline & One & $94(11.7)$ \\
\hline & Two to four & $313(38.8)$ \\
\hline & $\geq$ Five & $299(37.1)$ \\
\hline \multirow[t]{3}{*}{ Type of last delivery } & Normal delivery & $561(69.6)$ \\
\hline & Caesarian section & $114(14.1)$ \\
\hline & Vacuum/forceps & $131(16.3)$ \\
\hline \multirow[t]{3}{*}{ Sex of attendant on delivery } & Male & $264(32.8)$ \\
\hline & Female & $434(53.8)$ \\
\hline & Both & $108(13.4)$ \\
\hline \multirow[t]{4}{*}{ Type of last delivery } & Normal delivery & $561(69.6)$ \\
\hline & Caesarean delivery & $113(14.0)$ \\
\hline & Instrumental delivery & $18(2.2)$ \\
\hline & Delivery by episiotomy & $114(14.1)$ \\
\hline \multirow[t]{2}{*}{ Facing complication during labour and delivery } & No & $651(80.8)$ \\
\hline & Yes & $155(19.2)$ \\
\hline
\end{tabular}

gave birth five or more children, of which 69.6\% had SVD; $53.8 \%$ were attended by female attendant on delivery. Nearly three quarter $(72.3 \%)$ of study participants had attended ANC for the index pregnancy and the majority $(87.8 \%)$ of them had less than four ANC follow up.

\section{Prevalence of compassionate and respectful maternity care}

The overall prevalence of disrespect and abuse during child birth in this study was $63.5 \%(95 \% \mathrm{Cl}$ : 60.2 67.2). As indicated in Figure 1, the most common type of disrespect and abuse mentioned by study participants were; non-confidential care $(50.5 \%)$; non-consented care $(46.5 \%)$, physical abuse (34.5\%) and denial of care (33.0\%).

\section{Factors associated with disrespect and abuse during health facility child birth}

In the bi-variable binary logistic regression analysis, age of mother in years, mothers' level of education, family monthly income (ETB), number of ANC visit for last pregnancy, parity, mode of current delivery, long stay at the health facility and facing complication during labour and delivery had a P-value $<0.25$ and they were consider as candidate for multivariable binary logistic regression analysis.

After controlling for confounders in the multivariable analysis; the respondents' level of education, number of ANC visit for current pregnancy, mode of current delivery, and complications faced during labour and delivery had significant association with disrespect and abuse. Mothers who had no formal education were 1.65 times $(A O R=1.645 ; 95 \% \mathrm{Cl}: 1.070,2.531)$ and primary (1-8) education were 2.6 times, $(\mathrm{AOR}=2.626 ; 95 \% \mathrm{Cl}$ : $1.691,4.079)$ more likely to experience disrespect and abuse than those educated secondary and above level.

Similarly, women who attended ANC for less than 4 visits for current pregnancy had 1.75 times more likely to be disrespected and abused $(\mathrm{AOR}=1.753 ; 95 \% \mathrm{Cl}: 1.263$, 2.431) when compared with mothers who had greater than 4 number of ANC visit for their current pregnancy. Those respondents who gave birth by SVD were 2.3 times more likely to be disrespected and abused (AOR 


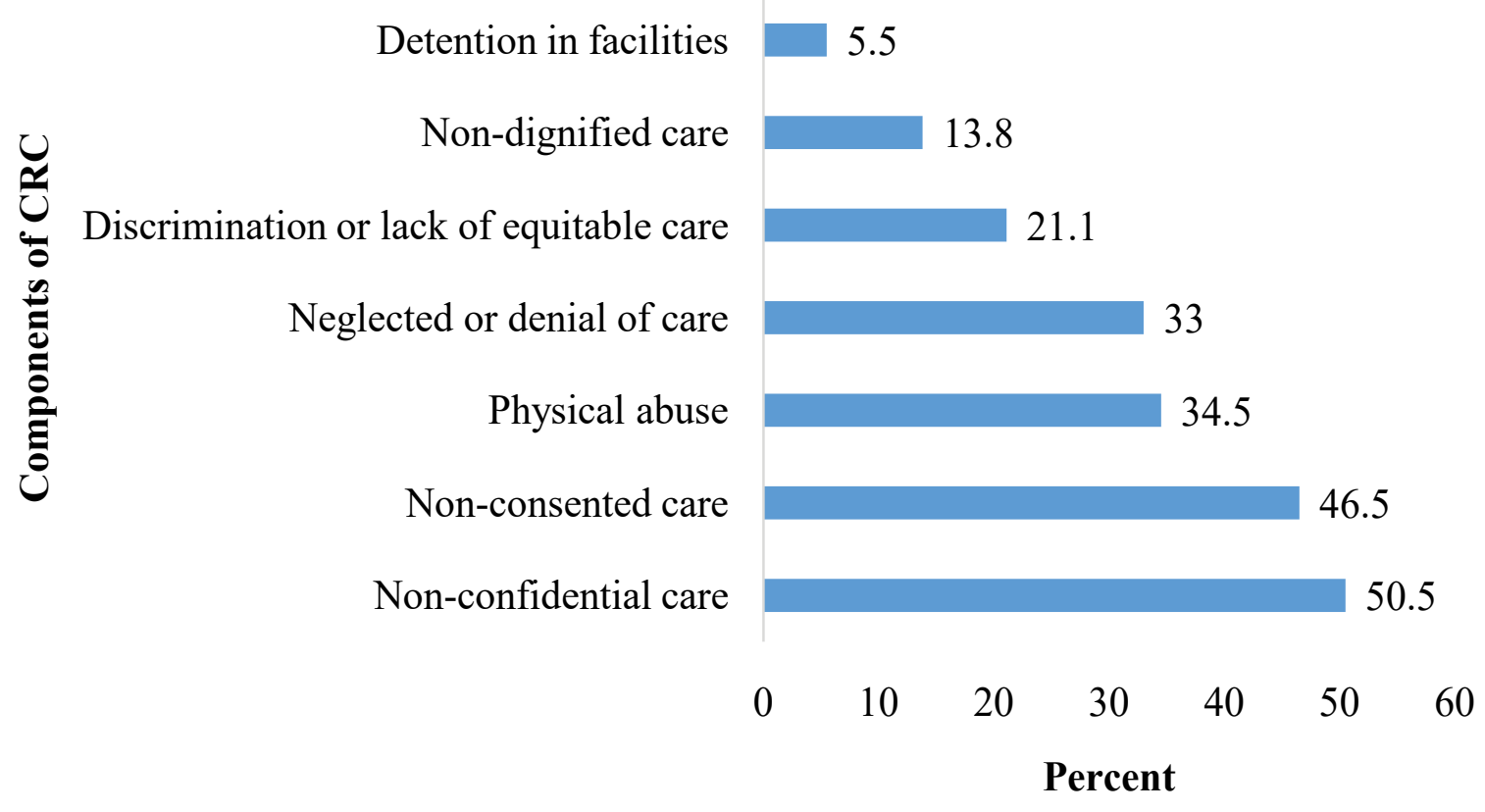

Figure 1: The prevalence of components of CRMC among women who gave birth in public health facility of Hawassa City, 2019.

Table 3: Bivariable and multivariable logistic regression analysis for disrespect and abuse among women who gave birth in public health facility of Hawassa City, 2019.

\begin{tabular}{|c|c|c|c|c|c|}
\hline & \multicolumn{2}{|c|}{ Disrespect and Abuse } & \multirow[t]{3}{*}{ COR $(95 \% \mathrm{Cl})$} & \multirow[t]{3}{*}{ AOR (95\% Cl) } & \multirow[t]{3}{*}{ P-Value } \\
\hline & Yes & No & & & \\
\hline & No. (\%) & No. (\%) & & & \\
\hline \multicolumn{6}{|l|}{ Age of mother in years } \\
\hline $15-24$ & $155(69.5)$ & $68(30.5)$ & $1.51(1.03,2.2)$ & $2.04(0.94,4.41)$ & 0.07 \\
\hline $25-34$ & $201(62.0)$ & $123(38.0)$ & $1.08(0.77,1.51)$ & $1.56(0.85,2.83)$ & 0.15 \\
\hline 35 and above & $156(60.2)$ & $103(39.8)$ & 1 & 1 & \\
\hline \multicolumn{6}{|c|}{ Mothers level of education } \\
\hline No formal education & $158(62.5)$ & $95(37.5)$ & $1.52(1.05,2.19)$ & $1.64(1.07,2.53)$ & $0.02^{*}$ \\
\hline Primary (1-8) & $239(71.8)$ & $94(28.2)$ & $2.32(1.63,3.32)$ & $2.63(1.69,4.08)$ & $<0.01^{*}$ \\
\hline Secondary and above & $115(52.3)$ & $105(47.7)$ & 1 & 1 & \\
\hline \multicolumn{6}{|c|}{ Family monthly income (ETB) } \\
\hline$<1000$ ETB & $140(69.7)$ & $61(30.3)$ & $1.7(1.08,2.68)$ & $1.47(0.86,2.5)$ & 0.16 \\
\hline 1000-2575 ETB & $295(62.6)$ & $176(37.4)$ & $1.24(0.84,1.83)$ & $1.19(0.75,1.88)$ & 0.46 \\
\hline$>2575$ ETB & $77(57.5)$ & $57(42.5)$ & 1 & 1 & \\
\hline \multicolumn{6}{|c|}{ No of ANC visit for current pregnancy } \\
\hline$<4$ & $353(68.9)$ & $159(31.1)$ & $1.88(1.40,2.54)$ & $1.75(1.26,2.43)$ & $0.01^{*}$ \\
\hline$\geq 4$ & $159(54.1)$ & $135(45.9)$ & 1 & 1 & \\
\hline \multicolumn{6}{|l|}{ Parity } \\
\hline Nullipara & $66(66.0)$ & $34(34.0)$ & $1.23(0.76,1.98)$ & $1.12(0.58,2.17)$ & 0.74 \\
\hline One & $68(72.3)$ & $26(27.7)$ & $1.66(1.00,2.76)$ & $1.00(0.47,2.16)$ & 1.00 \\
\hline Two to four & $195(62.3)$ & $118(37.7)$ & $1.05(0.76,1.45)$ & $2.121(0.91,4.96)$ & 0.08 \\
\hline$\geq$ Five & $183(61.2)$ & $116(38.8)$ & 1 & 1 & \\
\hline \multicolumn{6}{|l|}{ Mode of current delivery } \\
\hline Normal delivery & $385(68.6)$ & $176(31.4)$ & $2.36(1.60,3.47)$ & $2.35(1.48,3.72)$ & $<0.01^{*}$ \\
\hline Caesarian section & $64(56.1)$ & $50(43.9)$ & $1.38(0.83,2.29)$ & $1.40(0.80,2.46)$ & 0.24 \\
\hline Vacuum/forceps & $63(48.1)$ & 68 (51.9) & 1 & 1 & \\
\hline
\end{tabular}




\begin{tabular}{|l|l|l|l|l|l|}
\hline Yes & $359(66.7)$ & $179(33.3)$ & $1.51(1.12,2.04)$ & $1.1(0.71,1.7)$ & 0.67 \\
\hline No & $153(57.1)$ & $115(42.9)$ & 1 & 1 & \\
\hline Facing complication during labour and delivery & & & $1.6(1.04,2.46)$ & $0.03^{*}$ \\
\hline Yes & $113(72.9)$ & $42(27.1)$ & $1.7(1.15,2.50)$ & 1 & \\
\hline No & $399(61.3)$ & $252(38.7)$ & 1 & \\
\hline
\end{tabular}

NB; ${ }^{*} P$-value $<0.05$ was statistically significant on multivariable, $1=$ Reference.

$=2.347 ; 95 \% \mathrm{Cl}: 1.482,3.716)$ as compared with those managed by instrumental delivery. Participants who faced complication during labour and delivery were 1.6 times more likely to be disrespected and abused $(\mathrm{AOR}=1.60 ; 95 \% \mathrm{Cl}: 1.04,2.46)$ than those who did no experience of any complication during labour and delivery (Table 3).

\section{Discussion}

This study examined the compassionate and respectful maternity care and associated factors among women attending delivery at different health institutions. Overall, nearly two third of respondents reported as they faced at least one form of disrespect and abuse during childbirth. Factors identified to have association with disrespect and abuse during health facility child birth were, educational status of the woman, number of ANC visit for current pregnancy, mode of current delivery, and complications faced during labour and delivery.

In this study, the overall prevalence of disrespect and abuse during health facility child birth was $63.5 \%$ (95\% $\mathrm{Cl}$ : 60.2-67.2). This finding is in line with study conducted in Bahir Dar town, Ethiopia 67.1\% [26]. This finding was lower than the findings of studies in Addis Ababa and Western Oromia [14,21], however, it was very high in relation to the finding of the study conducted to assess the quality of care for prevention and management of common maternal and newborn complications in hospitals of Ethiopia [27]; Therefore studies direct to prevent disrespect and abusive maternity to increase skilled birth attendance that could humper the maternal and neonatal mortality [28].

Inversely, $36.5 \%$ were managed by culturally appropriate way as per the standard of compassionated and respected care which was insignificant in comparison to the finding (90.8\%) of Addis Ababa study [14]. This is very dependent on knowledge and skill of health care providers about compassionate care [29] and the presence or absence of companion in delivery room [30-32]. This strongly encourage interventions that save the lives of both mother and her child and also promote ensuring continuity of care during birth to achieve universal health coverage [24,33,34]. Our study identified that $13.3 \%$ were separated from baby without medical indication, that is very high in comparison to finding (2.3\%) of study in Addis Ababa [14]. This is one of women unfriendly care $[5,35]$ in which women express they were not satisfied on the care obtained to them and it does not promote immediate breast feeding initiation [31,36].

However, WHO promotes non denial of access to life-saving essential obstetric care when complications occur during pregnancy or childbirth [37] because all women have the right to timely health care and to the highest attainable level of health [38]. In this $13.3 \%$ of study participants were denied from food or fluid in labor unless medically necessitated, it deemed to be lower than the finding of report on countries experience [38]. Perhaps, this could be lack strong standard that enforce health care providers to act to maximum effort in Ethiopia [39].

In this study, $37.3 \%$ of women were deprived privacy when they were in second stage of labour which could be lack of curtain or separate room $[19,31,39,40]$. For instance, the care providers never used drapes to control infection. Non-confidential care is an especially important problem in which the discrimination a woman experiences in a facility and her community and act to deter her use of facility-based childbirth care $[5,41]$.

However, World health organization stated that obtaining consent and ensuring confidentiality are one of the universal ethical standards in quality of health care [37], and these are key concepts to ensure compassionated care $[40,42]$, in our study, the vast majority of study participants were treated with nonconfidential care and not obtained consent before care which very high in comparison to findings of studies conducted elsewhere (only 2-8.5\%) of women experienced non-confidential care [11-13]. But the level of consent obtained was consistent to finings of different studies [26,38]; besides, abandonment, one of indication of deficiencies in respectful care for laboring women, was the frequently mentioned type of disrespect and abuse in many studies $[19,40]$. There is a probable correlation of results in between countries. For instance, our study identified that $40.2 \%$ of women were ignored or abandoned the service when they need, which is also in line with the studies of elsewhere $[9,38]$ but it was a little bit higher than the study conducted in Addis Ababa [14].

The result of our study identified that shortage of infrastructure, high client/patient flow, and/ or late referring system, that could determine the compassionated care to women in labor and in turn affect the quality maternity care was similar to findings of studies [11,21-23,34,37,38]; moreover in this study, 
burn out of care providers, weak health institution management system, poor updating of both skill and knowledge and lack of carrier development among care providers were identified problems as determining factors for disrespected and abusive maternity care among labouring women because these could alter the view/behaviour of care providers including stress $[33,37,43-45]$, and supported by many studies in globe $[9,19,22-24,34]$.

Though the study was observational, it was proposed to be relatively rapid and not a detailed examination of all aspects of compassionate care. Lack of qualitative data, and associated factors were not exhaustibly explored in each interview steps. This limited our capacity to reasonably present the relative importance and alternative solution for resolution of dis-compassionate aspect of care and acculturalization of disrespect maternal care.

\section{Conclusion}

Non-compassionated care is one of fundamental barrier of skilled birth attendance. The prevalence of Non-compassionated care during childbirth was found to be high and it was more prevalent in women with no formal education and primary education and low ANC follow up. Woman-centered care in a respectful and non-abusive manner can be promoted by addressing identified factors, creation of awareness, strengthening health management strategies through capacitating institutional structure and updating professional through ongoing in-service and academic trainings are proposed solutions. However, further follow up studies could differentiate the underlining causes.

\section{Acknowledgements}

Above all, we praise Almighty God for He has strengthened our hands, never set us aside in all our ups and downs, and during our happiness and sorrow times, and despite all other constraints, brought me to this time. Next, we would like to express our heartfelt gratitude to Pharma Health Sciences College of Hawassa Campus, SNNP Regional Health Bureau and Hawassa City Administration of Health Office department for providing technical support to fulfill of our work and who have directly or indirectly supported in the process of the preparation of this study. The last but not the least, we would like to thank all those who provided constructive comments for success of this study.

\section{References}

1. WHO (2016) World health statistics 2016: Monitoring health for the SDGs, sustainable development goals. World Health Organization.

2. Central Statistical Agency (CSA), ICF (2016) Ethiopia demographic and health survey 2016. Addis Ababa, Ethiopia, and Rockville, Maryland, USA.

3. Madhiwalla N, Ghoshal R, Mavani P, Roy N (2018)
Identifying disrespect and abuse in organisational culture: A study of two hospitals in Mumbai, India. Reprod Health Matters 26: 36-47.

4. Grilo Diniz CS, Rattner D, Lucas d'Oliveira AFP, de Aguiar JM, Niy DY (2018) Disrespect and abuse in childbirth in Brazil: Social activism, public policies and providers' training. Reprod Health Matters 26: 19-35.

5. Tamara WM (2013) A guide for advocating for respectful maternity care. White Ribbon Alliance, USAID.

6. Solnes Miltenburg A, van Pelt S, Meguid T, Sundby J (2018) Disrespect and abuse in maternity care: Individual consequences of structural violence. Reprod Health Matters 26: 88-106.

7. WHO (2015) The prevention and elimination of disrespect and abuse during facility-based childbirth. World Health Organization.

8. Federeral Ministry of Health (2017) Health sector tansformation plan 2.

9. Abuya T, Warren CE, Miller N, Njuki R, Ndwiga C, et al. (2015) Exploring the prevalence of disrespect and abuse during childbirth in Kenya. PLoS One 10: e0123606.

10. Biresaw W (2017) Level of disrespect and abuse of women and associated factors during facility based childbirth in Bahir Dar Town, Amhara Regional State, Ethiopia.

11. Sando D, Ratcliffe $H$, McDonald K, Spiegelman D, Lyatuu $\mathrm{G}$, et al. (2016) The prevalence of disrespect and abuse during facility-based childbirth in urban Tanzania. BMC Pregnancy Childbirth 16: 236.

12. Afulani PA, Aborigo RA, Walker D, Moyer CA, Cohen S, et al. (2019) Can an integrated obstetric emergency simulation training improve respectful maternity care? Results from a pilot study in Ghana. Birth 46: 523-532.

13. Warren C, Njuki R, Abuya T, Ndwiga C, Maingi G, et al. (2013) Study protocol for promoting respectful maternity care initiative to assess, measure and design interventions to reduce disrespect and abuse during childbirth in Kenya. BMC Pregnancy Childbirth 13: 21.

14. Anteneh A, Delayehu B (2015) Status of respectful and nonabusive care during facility-based childbirth in a hospital and health centers in Addis Ababa, Ethiopia. Reprod Health 12: 33.

15. O'Connor M, McGowan K, Jolivet RR (2019) An awarenessraising framework for global health networks: Lessons learned from a qualitative case study in respectful maternity care. Reprod Health 16: 1.

16. Gebremichael MW, Worku A, Medhanyie AA, Berhane $Y$ (2018) Mothers' experience of disrespect and abuse during maternity care in northern Ethiopia. Glob Health Action 11: 1465215.

17. Anteneh A, Delayehu B, Alison M, Michelle K (2018) Service providers' experiences of disrespectful and abusive behavior towards women during facility based childbirth in Addis Ababa, Ethiopia. Reprod Health 15: 4.

18. LeFevre AE, Scott K, Mohan D, Shah N, Bhatnagar A, et al. (2019) Development of a phone survey tool to measure respectful maternity care during pregnancy and childbirth in India: Study protocol. JMIR Res Protoc 8: e12173.

19. Rosen HE, Lynam PF, Carr C, Reis V, Ricca J, et al. (2015) Direct observation of respectful maternity care in five countries: A cross-sectional study of health facilities in East and Southern Africa. BMC Pregnancy Childbirth 15: 306. 
20. Austad K, Chary A, Martinez B, Juarez M, Martin YJ, et al. (2017) Obstetric care navigation: A new approach to promote respectful maternity care and overcome barriers to safe motherhood. Reprod Health 14: 148.

21. Bobo FT, Kasaye HK, Etana B, Woldie M, Feyissa TR (2019) Disrespect and abuse during childbirth in Western Ethiopia: Should women continue to tolerate? PLoS One 14: e0217126.

22. Sheferaw ED, Mengesha TZ, Wase SB (2016) Development of a tool to measure women's perception of respectful maternity care in public health facilities. BMC Pregnancy Childbirth 16: 67

23. Banks KP, Karim AM, Ratcliffe HL, Betemariam W, Langer A (2018) Jeopardizing quality at the frontline of healthcare: Prevalence and risk factors for disrespect and abuse during facility-based childbirth in Ethiopia. Health Policy Plan 33: 317-327.

24. Shiferaw S, Spigt M, Godefrooij M, Melkamu Y, Tekie M (2013) Why do women prefer home births in Ethiopia? BMC Pregnancy Childbirth 13: 5.

25. Webber G, Chirangi B, Magatti N (2018) Promoting respectful maternity care in rural Tanzania: Nurses' experiences of the "Health Workers for Change" program. BMC Health Serv Res 18: 658.

26. Wassihun B, Deribe L, Worede N, Gultie T (2018) Prevalence of disrespect and abuse of women during child birth and associated factors in Bahir Dar town, Ethiopia. Epidemiol Health 40: e2018029.

27. Getachew A, Ricca J, Cantor D, Rawlins B, Rosen H, et al. (2011) Quality of care for prevention and management of common maternal and newborn complications: A study of Ethiopia's hospitals. Baltimore, Maryland, USA.

28. WHO (2014) The prevention and elimination of disrespect and abuse during facility-based childbirth. World Health Organization, Geneva, Switzerland.

29. PATH (2002) Violence against Women: Effects on Reproductive Health. Seattle, Washington.

30. Ndwiga C, Warren C, Abuya T, Kanya L, Maranga A (2014) Respectful Maternity Care Resource Package. In: Council P, Community Facilitator's Guide. Population Council, New York.

31. International Federation of Gynecology and Obstetrics, International Confederation of Midwives, White Ribbon Alliance, International Pediatric Association, World Health Organization, et al. (2015) Mother-baby friendly birthing facilities. Int J Gynaecol Obstet 128: 95-99.
32. FIGO (2014) Mother and newborn friendly birthing facility.

33. Behruzi R (2011) What are the components of humanized childbirth in a highly specialized hospital? An Organizational Case Study. Université de Montréal.

34. USAID (2015) Ending preventable maternal mortality: USAID maternal health vision for action evidence for strategic approaches. Washington, DC.

35. USAID, Woman friendly care: A guide.

36. Martin CJH (2014) The Birth Satisfaction Scale (BSS). Midwifery Matters 3.

37. WHO U, UNFPA (1999) Women-friendly health services experiences in maternal care: Report of a Workshop. Mexico City.

38. Veronica R, Barbara D, Catherine C, Jeffrey S (2012) Respectful maternity care, country experiences: Survey report.

39. Ramsey M (2012) Ethiopia gender assessment. Chapel Hill, NC.

40. Bohren MA, Vogel JP, Hunter EC, Lutsiv O, Makh SK, et al. (2015) The Mistreatment of Women during Childbirth in Health Facilities Globally: A Mixed-Methods Systematic Review. PLoS Med 12: e1001847.

41. Bowser D, Hill K (2010) Exploring evidence for disrespect and abuse in facility-based childbirth: Report of a landscape analysis. Harvard School of Public Health, University Research Co., LLC.

42. National Institute for Health and Clinical Excellence: Guidance (2007) Intrapartum care: Care of healthy women and their babies during childbirth. RCOG Press, London.

43. Peabody JW, Taguiwalo MM, Robalino DA, Frenk J, Jamison DT, et al. (2006) Improving the quality of care in developing countries. In: Disease Control Priorities in Developing Countries. ( $2^{\text {nd }}$ edn), The International Bank for Reconstruction and Development/The World Bank, Washington (DC).

44. Goldstein WHaM (2012) "Being treated like a human being": Attitudes and behaviours of reproductive and maternal health care providers.

45. Karkee R, Lee AH, Pokharel PK (2014) Women's perception of quality of maternity services: A longitudinal survey in Nepal. BMC Pregnancy Childbirth 14: 45. 